\title{
Presentación. La construcción de la antropología en los países andinos
}

\author{
Presentation. The construction of anthropology in the Andean countries
}

Eloy Gómez Pellón

Catedrático de Antropología Social. Universidad de Cantabria. Santander (España)

gomezel@unican.es

\section{LA CONSTRUCCIÓN DE LA ANTROPOLOGÍA EN LOS PAÍSES ANDINOS MONOGRÁFICO COORDINADO POR ELOY GÓMEZ PELLÓN}

\section{RESUMEN}

El presente monográfico constituye una exploración de la forma en que se ha construido la antropología en los países andinos. Todos ellos configuran un área geográfica definida por la marcada personalidad que le proporciona la cordillera andina. También están unidos por razones históricas, tanto precoloniales como coloniales y republicanas. Algunos de ellos, como son los casos de Colombia, Venezuela y Ecuador, formaron parte de aquel Estado que la historiografía ha denominado la Gran Colombia, cuyo componente andino era muy notable. Además, Ecuador, Perú y Bolivia poseen altos porcentajes de población indígena que explica su fuerte diversidad cultural. En los casos de Colombia y Venezuela, esta población indígena es reducida, pero son países donde la diversidad cultural es, igualmente, muy marcada, debido a la existencia de una notable presencia de afrodescendientes, especialmente en las áreas vinculadas a los monocultivos comerciales que eran las plantaciones. En definitiva, son países que además de compartir geografía en buena medida y de poseer una historia imbricada, vivieron el despertar de una antropología enfocada al estudio de sus numerosas culturas. Sin embargo, la construcción de la antropología ha sido heterogénea, producto de una compleja institucionalización.

\section{ABSTRACT}

This monograph is an exploration of the way anthropology has been constructed in the Andean countries. All of them make up a geographical area defined by the marked personality that the Andean mountain range provides. They are also united for historical reasons, both pre-colonial and colonial and republican. Some of them, such as the cases of Colombia, Venezuela and Ecuador, were part of that state that historiography has called Greater Colombia, whose Andean component was very notable. In addition, Ecuador, Peru and Bolivia have high percentages of indigenous population that explains their strong cultural diversity. In the cases of Colombia and Venezuela, this indigenous population is small, but they are countries where cultural diversity is also very marked, also due to the existence of a notable presence of Afro-descendants, especially in areas linked to monocultures commercial that were the plantations. In short, they are all countries that, in addition to sharing geography to a large extent and having an overlapping history, experienced the awakening of an anthropology focused on the study of their many cultures. However, the construction of anthropology has been heterogeneous, the product of a complex institutionalization.

PALABRAS CLAVE

antropologías latinoamericanas | antropologías andinas |antropologías del Sur | antropología crítica latinoamericana | nuevas antropologías KEYWORDS

Latin American anthropologies | Andean anthropologies | anthropologies of the South | Latin American critical anthropology | new anthropologies

\section{Introducción}

Los países andinos, que descubrieron pronto el interés por los temas propios de la antropología, tardaron tiempo en institucionalizar la disciplina, y aún más tiempo en generar una reflexión sobre las antropologías que estaban construyendo. Ciertamente, cada uno de estos países posee su propia tradición, y, si bien las diferencias entre ellos son muy acusadas, comparten un fondo común de experiencias y de preocupaciones, así como de redes de conocimiento. Aunque en algunos de ellos, como Perú, Colombia y Venezuela, la institucionalización se produjo en los años cuarenta del siglo XX, y en otros, como Ecuador y Bolivia, esta se retrasó hasta los años setenta de ese mismo siglo, en todos 
ellos se ha producido una evolución, comparable desde muchos puntos de vista, debida tanto a la pluralidad de estos Estados como a las interacciones que, por razones históricas, pero también desde la perspectiva académica e investigadora, se han producido entre estos países.

En todos los países andinos la antropología ha sido elaborada tanto por autores nacionales como por los autores extranjeros que desarrollan o han desarrollado su quehacer investigador, y ocasionalmente docente, en dichos países. Aunque estos últimos han sido comparativamente una minoría, su influencia ha sido extraordinaria, especialmente en los casos de los antropólogos franceses y norteamericanos. A su vez, también estos antropólogos extranjeros han sido sensibles, con mucha frecuencia, a las preocupaciones de los antropólogos nativos. Además, han sido numerosos los antropólogos de los países andinos, desde los años cuarenta del siglo XX, que han adquirido su formación en Francia, Reino Unido y los Estados Unidos, lo cual explica que se trate de antropologías nutridas con planteamientos teóricos y metodológicos de la antropología clásica, aunque dotadas de una personalidad propia, encuadrable en el ámbito latinoamericano, y más concretamente en la tradición disciplinar de cada país. Indudablemente, no existe una antropología latinoamericana ni una antropología andina, sino muchas antropologías, incluso dentro de los propios Estados nacionales. A la complejidad del proyecto político de cada Estado se han sumado el delicado papel con el que cumple la antropología en países muy necesitados del conocimiento de la realidad pluricultural y plurinacional.

\section{Diversidad de antropologías}

Dicho esto, se puede añadir que existen tradiciones locales y regionales, diferenciadas hasta cierto punto de la antropología más al uso en Europa y en los Estados Unidos, unidas por el común denominador de la resistencia frente a estas últimas, lo cual ha llevado a pensar a algunos que se trata de unas antropologías con particularidades epistemológicas y metodológicas, que podrían situarnos ante una forma distinta de construcción de la antropología. Fue a finales de los años sesenta del siglo XX y primeros años setenta cuando se produjeron los primeros cuestionamientos de una antropología tenida por occidental, e incluso por colonizadora, para la cual se ha reservado posteriormente una pléyade de denominaciones al uso, que por lo general encierran juicios de valor (antropología hegemónica, antropología dominante, antropología metropolitana, antropología burguesa, etc.) y solo en ocasiones denominaciones con aspiraciones de neutralidad relativa (antropología originaria, antropología del Norte, antropología primera, antropología clásica, etc.). Bajo estas denominaciones late la consideración de que nos hallamos ante una antropología surgida inicialmente al calor del colonialismo, con la intención de contribuir al conocimiento de los nativos, y al mismo tiempo administrados, bajo consideraciones epistemológicas y metodológicas que podrían haber resultado viciadas por el fin del conocimiento que se establecía.

Se genera así una concepción binomial de la antropología, de acuerdo con la cual, frente a esta antropología hegemónica son utilizadas muy diversas denominaciones, si bien por lo regular formuladas en plural debido a la inmensa variedad de puntos de vista: antropologías segundas, antropologías subalternas, antropologías nativas, y, quizá, sobre todo, antropologías del Sur, o del Sur global. Otras denominaciones, como la de antropología indígena, han resultado desplazadas progresivamente (vid. Angosto 2015). De esta manera se da a entender que no existe una antropología que unitariamente de respuesta a la antropología arraigada en Europa y Norteamérica, sino muchas antropologías locales. Se produce así una asignación de nombres que no son sino trasunto de los innumerables dualismos utilizados en la clasificación mundial de las sociedades, esto es, desarrollo y subdesarrollo, primer mundo y tercer mundo, Norte y Sur, dominación y dependencia, núcleo y periferia, modernidad y tradición, etc., como ha explicado E. Krotz $(1993,2015)$. Se quiere poner de manifiesto de este modo, o al menos así sucedió durante un tiempo por parte de algunos, que existen muchas antropologías, dicho unas veces de una manera más radical y otras veces más atenuada.

Estas antropologías del Sur se sustentarían en la idea de un cambio en la manera de entender la alteridad. Las antropologías del Sur, lejos de ver al otro como distinto del sujeto cognoscible, y, al mismo 
tiempo distante, han defendido la cercanía del investigador y el sujeto de conocimiento en el caso de las sociedades de América Latina, y, en general, en el de todas las que nacieron como resultado de un proceso de descolonización, más cercano o más lejano en el tiempo, que, en el caso de América Latina es inseparable de lo que Quijano (2000) y otros han denominado colonialidad, en alusión al canon clasificador de las personas que inflama la desigualdad social. Ciertamente, la descolonización abocó al nacimiento de Estados nacionales caracterizados por la invisibilidad y la exclusión de poblaciones que ni siquiera ha cesado en el presente. Los antropólogos, de acuerdo con esta reflexión, no solo son, muy a menudo, miembros de esas sociedades, sino que su vivencia cotidiana no podría entenderse si no fuera como parte de una cotidianidad conflictiva, en la que los prejuicios, los estereotipos, la discriminación y la desigualdad son las monedas de cambio. En estas condiciones, el otro que estudia el antropólogo no es realmente uno distinto sino que es uno mismo, encarnado en la vida de los estudiados. Por lo tanto, la teoría de la reflexión que se encuentra tras las antropologías del Sur comporta un cuestionamiento de esa otra alteridad que ha sido distintiva de la ciencia antropológica desde su mismo nacimiento.

Progresando en la crítica a la antropología clásica, se ha dicho en más de una ocasión, sobre todo en los años setenta y ochenta del siglo pasado, que la misma no era idónea para el estudio de las sociedades excluidas o marginadas. De este modo, la antropología nativa constituiría una ruptura con la epistemología tradicional, e incluso con la ciencia occidental, considerada como brazo de un conocimiento que, a mayores, ha sido tildado de liberal, burgués y capitalista. Esta sería la posición más extrema, producto de una respuesta radical y extraordinariamente crítica. Se dice, con mucha frecuencia, que esta antropología nativa representa un nuevo paradigma superador de los existentes. A su lado, otras percepciones, más moderadas, conciben la existencia en Latinoamérica de una antropología que bien puede calificarse de alternativa a la clásica o de complementaria de la misma. Todas estas propuestas, que sin duda tienen su interés, muestran una adhesión heterogénea hacia las antropologías nativas, en las que se pone de manifiesto el deseo de mostrar la personalidad de las antropologías locales, en otro tiempo opacadas por la antropología más clásica y dualista, en la que el sujeto de conocimiento era un otro distante, al que se acercaba el sujeto cognoscente con ánimo de curiosidad y con el deseo de realizar una observación. Durante la observación se creaba la ficción de una proximidad, que tan solo duraba el tiempo durante el cual se producía el trasvase de la información y que servía para seguir construyendo la antropología en su sentido más clásico.

La nueva manera de entender la relación entre el observador y los observados, en un sector la antropología latinoamericana, habría generado una sensación de crisis. Sin embargo, Roberto Cardoso $(1988,1996)$ estuvo siempre convencido de que no existía tal crisis de la antropología en Latinoamérica, y menos aún una crisis de los modelos explicativos que afectara a los aspectos paradigmáticos o a los epistemológicos. La verdad es que lo contrario parece, más bien, de una hipérbole, sin apenas visos de verosimilitud. Ciertamente, como extraordinario conocedor de la antropología latinoamericana, su afirmación parece difícilmente rebatible. Él siguió con mucha frecuencia una obra que ha sido libro de cabecera de muchos de nosotros. Me estoy refiriendo a la de Kuhn (1962), La estructura de las revoluciones científicas, y también a la utilidad para permitirnos entender el concepto de paradigma. Es obvio que se trata de un término manoseado hasta la saciedad, que significa tantas cosas en las ciencias sociales que, a veces, es difícil entenderle el sentido. Lo mismo se usa con el valor de sistema de ideas, que con el de corriente de pensamiento, procedimiento de investigación, enfoque, y con el de tantas y tantas cosas. Y, sin embargo, según el propio Kuhn, el paradigma es una perspectiva teórica, mucho más amplia que la teoría, que equivale a una visión del mundo. Hasta podríamos decir que los paradigmas son marcos teórico-metodológicos, que en las ciencias experimentales son muy cambiantes, al revés que en las sociales. Más aún, para aceptar que en las ciencias sociales existen los paradigmas debemos prescindir primero del requisito de que han de ser conocidos y admitidos por la comunidad científica entera. De hecho, Kuhn rebaja los requisitos del paradigma para que su noción pueda ser utilizada en las ciencias sociales, pero también insiste en que ello no puede devenir en banalización, considerando en todo caso que un paradigma no es una teoría, ni una corriente de pensamiento, ni un procedimiento de investigación.

Si de esa visión general de las ciencias sociales pasamos al caso de la antropología (vid. Gómez-Pellón 2012), enseguida nos percatamos que esta cuenta con unos pocos paradigmas propios, característicos y 
admitidos por los antropólogos como tal. Se pueden reducir al racionalista, de tradición francesa que devino en estructuralismo, al funcionalista, conectado con el anterior, de carácter anglosajón, al culturalista norteamericano, de raíz anglosajona y empirista, y, finalmente, al hermenéutico, vinculado a la filosofía alemana que da vida al interpretativismo. Precisamente, la reacción que se suscitó a comienzos del último cuarto del siglo pasado por parte de la antropología crítica latinoamericana hacia las escuelas en boga en las décadas precedentes, esto es, hacia las correspondientes al funcionalismo inglés, al estructuralismo francés y al culturalismo norteamericano, consistió en atribuirles a estas una falta de compromiso político y un notable desinterés por los problemas sociales, parapetadas tras una aparente objetividad, que lastraba los resultados. En definitiva, tal como explica $\mathrm{E}$. Restrepo, refiriéndose al caso latinoamericano en general (2016: 63-68) y al colombiano en particular (2014: 91-92), la aparente indiferencia de las escuelas tradicionales era percibida por los entusiastas de la antropología crítica como un apoyo nada desdeñable a los intereses de los sectores dominantes. No obstante, y esta es otra de las características de las ciencias sociales, como nos explica Kuhn, a menudo pueden convivir varios paradigmas a un mismo tiempo, de manera que la insatisfacción de una parte de los cultivadores de la antropología puede hacer que se erija uno nuevo que se une a los ya existentes. Al contrario, en las ciencias experimentales, parece que lo común es que, a medida que progresa la ciencia, un paradigma desplace a otro, provocando la muerte del precedente. En el ejemplo de Kuhn de la física se puede ver cómo, al modificarse el problema por el avance en la resolución, se reorienta la disciplina y se transforma la perspectiva teórica, es decir, la ventana para ver el mundo de la disciplina que es el paradigma. En la física óptica cuando Planck llevó a cabo la interpretación cuántica de la luz, se derrumbó la interpretación ondulatoria, que, a su vez, había anulado a la corpuscular. Evidentemente, no es el caso de la antropología, ni el de las ciencias sociales en general.

El propio Restrepo (2016), cuando traza el panorama de la antropología latinoamericana en estos años setenta y ochenta del siglo $\mathrm{XX}$, señala cómo el compromiso político se convirtió por entonces en la referencia insoslayable de una parte de los antropólogos, y tanto o más entre los estudiantes de antropología. El desdén por la percepción diacrónica de las sociedades americanas que mostraban algunas escuelas, afectas a los paradigmas más clásicos, las convirtió no solo en blanco de las críticas sino en objeto de rechazo. Por supuesto, los grupos indígenas, pero también las poblaciones campesinas, las de color, los barrios pobres de las ciudades, y poco a poco las mujeres, además de mostrar las huellas del pasado, carecían de un tratamiento específico por parte de las escuelas hegemónicas. Muchos de estos críticos tenían una visión revolucionaria de la vida que les hacía pensar en planteamientos que privilegiaban la dimensión histórica, los cuales resultaban más aptos para analizar las desigualdades y realizar propuestas transformadoras de la sociedad. Esta crítica de la antropología se suscitó con más virulencia allí donde existían universidades que enseñaban la disciplina, y donde había una masa de estudiantes y de profesores con la suficiente fuerza como para hacerse eco de las reivindicaciones sociales y proclamar la necesidad de un compromiso revolucionario.

De este modo, en Latinoamérica la antropología se ve repentinamente inmersa en un conflicto que, por otro lado, tuvo escasa repercusión en los ambientes académicos europeos y norteamericanos, salvo la que se mostró a nivel de individualidades. En los ambientes académicos latinoamericanos la repercusión fue algo mayor, pero sus efectos fueron escasos o moderados. Por supuesto que la antropología madura de las escuelas tradicionales, que se servía de los paradigmas al uso, prosiguió su camino incorporando aspectos que resultaban útiles en sus análisis. Roberto Cardoso (1996, 1999, 2000) puso de manifiesto que era muy posible que se estuvieran confundiendo los problemas de adaptación que presentaba la antropología en la periferia, motivados por la precariedad de los recursos en la enseñanza y en la investigación, con una auténtica crisis de la disciplina. La consecuencia, en palabras de Cardoso, es que existen estilos de antropología que se mostraban en una diversidad de maneras de entender la disciplina (Cardoso y Rubén 1995). Es cierto que, como ha explicado E. Krotz $(1997,2007)$ en más de una ocasión, el silenciamiento a que han sido sometidas las antropologías del Sur, tampoco ha ayudado mucho a visibilizar los problemas con los que esta se ha venido enfrentando. En este sentido, y a propósito, hace algunos años, Carlos Alberto Uribe (1997) concordaba en las apreciaciones de Krotz, pero dejando a salvo la condición de que toda antropología, sea la que fuere, debía conservar el derecho a mantener una personalidad propia y a singularizarse como tal frente a la antropología hegemónica. 
El mismo Krotz $(2007,2015)$ nos explica a propósito de la antropología, aunque su reflexión sirve para otros muchos campos, que no hay un solo Sur. En Europa, donde impera la antropología del Norte, también hay Sur, y las antropologías mediterráneas son un buen ejemplo, como ha explicado Susana Narotzki en el caso de España (2011), pero también las eslavas, balcánicas y otras. Son antropologías periféricas o "segundas", cuyos hallazgos no suelen ser especialmente tenidos en cuenta, ni sus antropólogos suelen ser seleccionados por los del Norte para entrar en diálogo con ellos. Son antropologías propias de ese Sur global, cuyas ciencias sociales en general, y cuyas antropologías en particular, suelen ser tan minusvaloradas. Los espacios del Sur han funcionado en el pasado como reservorios o espacios de colonización de las antropologías del Norte. En no pocas ocasiones se ha acusado a las antropologías del Norte (también en la Europa del Sur) de engrosar su corpus teórico en los países del Sur, y hasta de practicar una especie de colonialismo o neocolonialismo científico. No obstante, y a pesar de tantas contrariedades, puede ser cierto que la aportación de las antropologías del Sur a la teoría antropológica sea discreta. También puede ser debido a que las contribuciones nuevas en una disciplina solo adquieren relevancia cuando tienen la fuerza suficiente para derribar a las precedentes, puesto que, de lo contrario, corren el riesgo de quedar subsumidas en estas últimas, tal como explica Kuhn (1962) en su obra más clásica. Sin embargo, hay un problema superior puesto de relieve en multitud de ocasiones por Boaventura de Sousa (Sousa y Meneses 2014), y es el de las razones por las cuales la epistemología hegemónica ha prescindido de todos los saberes que podían contradecir su privilegiada posición. La pluralidad de saberes es una expresión más de la extraordinaria riqueza de las creaciones humanas, y por supuesto de las epistemologías de su conocimiento.

A menudo se ha dicho que fue de esta manera como la antropología crítica latinoamericana se aproximó al materialismo histórico, valiéndose de enfoques que trataban de indagar en las causas históricas de la desigualdad y el conflicto, y, por supuesto, en las consecuencias. Algunos antropólogos trataron de investigar cómo se había perpetuado en las sociedades latinoamericanas la desigualdad por razones étnicas y económicas, dando como resultado la permanente subordinación de los pueblos indígenas y de los grupos negros, y de una diversidad de colectivos desfavorecidos. Algunos trabajos, con perspectiva dialéctico-crítica, analizaron el funcionamiento de las relaciones de dominación y las estrategias que permiten a los grupos dominantes mantener sus posiciones de privilegio. Lejos de percibir en las estructuras sociales el todo armonioso que habían creído ver los funcionalistas y los funcionalestructuralistas, esta antropología crítica latinoamericana halló en estas estructuras la simiente del conflicto. En última instancia, esta perspectiva propugnaba la eclosión de una antropología que coadyuvara en la conquista del cambio revolucionario de la sociedad. Curiosamente, la teoría de la antropología dialéctico-crítica estaba anclada en la tradición europea, y ya por entonces había resultado cuestionada, tanto o más que otras antropologías tenidas por tradicionales, acaso porque el nuevo planteamiento no era menos tradicional que los cuestionados.

Con todo, la idea de que la cultura no es un ente independiente de otros ámbitos de la vida social, tal y como lo mostraba la antropología crítica latinoamericana, no era novedoso, sino que, más bien, es de admisión general que la cultura envuelve toda la vida social de los seres humanos. Sin embargo, los planteamientos de esta antropología latinoamericana enfatizaron el hecho de la inexistencia del conocimiento neutral. La antropología que producen los antropólogos, unas veces sin quererlo y otras queriéndolo, tiene un sesgo dado por la ideología, de forma análoga a lo que sucede en todas las ciencias sociales. Ello no contradice la idea de que el antropólogo, como cualquier científico social, investiga persuadido por el interés de hallar lo que, a la luz de los conocimientos existentes en la disciplina en ese momento, se estima como verdad. Es lo cierto que la antropología crítica latinoamericana no tuvo el peso que habría cabido esperar en la producción antropológica. Podríamos añadir que, en casos muy concretos, la radicalización de la antropología marxista dio lugar a planteamientos extremos que fueron rechazados por el resto de la antropología.

Simultáneamente, los antropólogos más afectos al cultivo de una antropología que podemos llamar clásica, continuaron realizando sus investigaciones y estableciendo sinergias con la antropología crítica, de suerte que se produjo un enriquecimiento de los temas característicos de la antropología. A finales del siglo XX el panorama antropológico se hallaba muy renovado, de suerte que el tratamiento de la alteridad había adquirido una gran riqueza. Las antropologías de los países andinos de finales del siglo XX y de 
los primeros lustros del siglo XXI revelan un gran interés por los colectivos étnicos, por el cambio cultural, por las políticas de desarrollo, y por una diversidad de temas a los que antes se les había prestado escasa atención, como los relativos a los afrodescendientes, las desigualdades de género, la educación, la salud, el medio ambiente (especialmente, los conflictos motivados por el aprovechamiento de los recursos extractivos), la interculturalidad, y otros, a los que se han añadido los derivados del postconflicto y de la memoria histórica.

Este enriquecimiento de la antropología, obviamente, no es exclusivo de Latinoamérica, pero es cierto que en esta parte del mundo adquiere caracteres propios. En la situación de la antropología en Latinoamérica a finales del siglo $X X$, se trató de buscar fórmulas que rebajaran la tensión, mediante la apertura de horizontes teóricos y metodológicos nuevos, trascendiendo los reduccionismos y los esencialismos. En el caso de la antropología colombiana, por ejemplo, entre finales del siglo $X X$ y comienzos del XXI, se genera una nueva coyuntura, que se conoce con el nombre de antropología en modernidad (Restrepo 2014), gracias a una concurrencia de voluntades, liderada por Instituto Colombiano de Antropología y algunas universidades, como la del Cauca, la Nacional y la de Los Andes), en la que colaboran también los antropólogos extranjeros que trabajan en Colombia ( $P$. Wade, J. Rappaport, C. Gros, A. M. Losonczy y otros), todos los cuales abogan por el giro discursivo, que, en este caso, fue de carácter postestructuralista. La explicación puede hallarse en que la antropología, después de todo, y como subrayaba Roberto Cardoso (1996: 10), siguiendo a Merleau-Ponty, es una forma de pensar, motivada por la interacción con la alteridad. De alguna manera, la antropología es una reflexión sobre el mundo social, de modo que la multiplicación de puntos de vista, lejos de frenar la dinámica la enriquece. Cuando hablamos de un paradigma relativamente novedoso y renovador en antropología pensamos en el interpretativismo de raíz hermeneuta (Geertz 1973, Gadamer 1960), el cual no se propuso jamás liquidar los existentes previamente, sino convivir con ellos para ensancharlos y enriquecerlos.

\section{La antropología en los países andinos}

El presente monográfico constituye una exploración de la forma en que se ha construido la antropología en los países andinos. Todos ellos configuran un área geográfica definida por la marcada personalidad que le proporciona la cordillera andina. También están unidos estos países por razones históricas, tanto precoloniales como coloniales y republicanas. Algunos de ellos, como son los casos de Colombia, Venezuela y Ecuador, formaron parte de aquel Estado que fue la Gran Colombia, cuyo componente andino era muy notable. Además, Ecuador, Perú y Bolivia poseen altos porcentajes de población indígena que explica su fuerte diversidad cultural. En los casos de Colombia y Venezuela, esta población indígena es reducida, pero son países donde la diversidad cultural es, igualmente, muy marcada, debido a la existencia de una notable presencia de afrodescendientes, especialmente en las áreas vinculadas a los monocultivos comerciales que eran las plantaciones. En definitiva, son países que además de compartir geografía en buena medida y de poseer una historia imbricada, vivieron el despertar de una antropología enfocada al estudio de sus numerosas culturas.

Sin embargo, la construcción de la antropología ha sido heterogénea, producto de una compleja institucionalización. Mientras que Perú, Colombia y Venezuela la lograron relativamente pronto, en Bolivia y Ecuador se retrasó considerablemente. En Perú esta institucionalización se evidenciará a comienzos de los años cuarenta, cuando, en 1941, debido a motivaciones endógenas y merced al entusiasmo de Luís E. Valcárcel, la Universidad de San Antonio Abad de Cuzco cuente con una sección de Historia y Antropología, antecedente de la implantación que se produce en la Universidad Mayor de San Marcos en 1945. El nacimiento del Instituto Francés de Estudios Andinos en 1948 y la fundación del Instituto de Estudios Peruanos en 1964, acelerarán la expansión de una antropología que iba a tener en la Universidad Mayor de San Marcos y, desde 1969, en la Pontificia Universidad Católica de Perú, sus centros de formación más poderosos. En la actualidad, Perú cuenta con una decena de programas de antropología, de los cuales tres se imparten en la capital (tras incorporarse la Universidad Nacional Federico Villarreal en 2003), y el resto se distribuyen por distintas universidades del país (Universidades 
de Cuzco, San Cristóbal de Huamanga, Centro del Perú, Trujillo, Arequipa, Puno y Amazonía Peruana), acogiendo un alumnado numeroso. El temprano nacimiento de la antropología peruana y la potencia de sus centros institucionales ha generado una vigorosa literatura andina, que se ha simultaneado tradicionalmente con otra amazónica de menor escala. Los artífices de esta abundante producción antropológica han sido los antropólogos nacionales y un reducido e influyente número de antropólogos extranjeros que, asimismo, contribuyeron a la institucionalización de la disciplina. En esa potente antropología andina, como explica Eloy Gómez Pellón, los sujetos antropológicos preferidos son las comunidades campesinas, resultando que su estudio espoleará poco a poco otros temas relacionados, como la etnicidad, el comunalismo, las formas de distribución, los bienes colectivos, las rondas campesinas y otros. Paralelamente, el objeto de estudio de la antropología amazónica residirá en las comunidades indígenas y en sus culturas. Conforme la antropología peruana ingresa en el siglo XXI, otros temas vertebrarán esta antropología, sin abandonar los precedentes: la educación, la salud, el género, la alimentación, la violencia y la memoria histórica, el extractivismo y otros, como explica el detallado análisis de Alejandro Díez Hurtado. En Perú hallamos una particularidad de la antropología, con respecto a la europea, que también la encontramos en el resto de los países andinos y, en general, en todos los de América Latina. Se trata de una doble vertiente de la antropología, que posee carácter investigador por un lado y profesional por otro, lo cual se proyecta sobre los intereses ocupacionales de sus graduados y postgraduados, proporcionando un gran vigor a la disciplina.

En Colombia el inicio de la institucionalización se produce en 1941, al mismo tiempo que en Perú, en torno al Instituto Etnológico Nacional, fundado por entonces bajo la dirección inicial de Paul Rivet, aunque habrá de esperarse al Primer Congreso Nacional de Antropología, en Popayán, en 1978, para que se produzca la auténtica eclosión de la antropología colombiana. Sin embargo, el primer programa universitario de antropología se había creado en fecha tan temprana como la de 1963, en la Universidad de los Andes de Bogotá, a la que le seguiría el de la Universidad Nacional en 1966, la cual contaba con una especialización antropológica en los estudios de Sociología desde 1959. Fuera de la capital, el proceso de institucionalización académica da lugar muy pronto a la creación de la titulación en la Universidad de Antioquia (1966) y en la del Cauca (1970). En la actualidad existe una quincena de programas de pregrado, de suerte que la mitad de éstos son de postgrado. La antropología colombiana actual es una antropología característica de las denominadas del Sur, esto es con acento, que como dicen Axel Rojas y Enrique Jaramillo, no trata de ser una antropología de las esencias, sino una antropología en diálogo, que contribuya a ofrecer una versión completa y rica del cultivo de la disciplina que se lleva a cabo en el país por parte de los antropólogos nacionales, pero también por la de los antropólogos extranjeros que han realizado y realizan sus investigaciones en Colombia. Se sigue así una tónica que está presente en otros países de América Latina y, cada vez más, en otras partes del mundo. Se trata de una antropología atenta a las innovaciones teóricas y metodológicas desde hace décadas, que acoge perspectivas y enfoques muy variados. Tan rica diversidad cultural y tan heterogéneo tratamiento, a modo de lo que podemos llamar pluralización, tiene su reflejo en este monográfico en un estudio de las relaciones interétnicas en el Chocó colombiano, que realiza Óscar Fernández Álvarez, en el cual se examina, en primer lugar, la escasa atención que ha recibido el territorio por parte de los antropólogos, y, en segundo lugar, cómo las comunidades negras e indígenas han creado un entorno compatible con la conservación natural, pero también con su propia supervivencia.

El inicio de la institucionalización de la antropología venezolana se produce también tempranamente. A pesar de la previa e intensa relación de la antropología norteamericana con el país, la primera institucionalización se produce cuando en 1949 se funda el Instituto de Antropología y Geografía, a impulso de Miguel Acosta Saignes, que sería el precedente del Instituto de Antropología e Historia ubicado en la Universidad Central de Venezuela. Saignes se había formado en la ENAH mexicana y realiza una siembra muy notable en Venezuela, al tiempo que aporta una perspectiva marxista, desconocida por entonces, a la antropología venezolana. Muy poco tiempo después, en 1952, George Hill, sociólogo norteamericano crearía el Departamento de Sociología y Antropología en la Facultad de Economía de la misma Universidad Central, contando con un grupo de antropólogos norteamericanos afectos al culturalismo. Casi al mismo tiempo nace el Instituto Caribe de Antropología y Sociología de la Fundación La Salle (1953), de modo que la antropología dispuso desde entonces con un andamiaje 
estructural que se fue afirmando según avanzaba el siglo $X X$, aunque pasando por etapas muy diversas, tal como nos explica Elizabeh Manjarrrés. En los últimos lustros del siglo XX la antropología se fortalece en la capital y se expande por la parte occidental (Mérida y el Zulia), hasta llegar en el presente a contar con ocho titulaciones, de las cuales dos son de licenciatura, tres de maestría y tres de postgrado. Aparte del Instituto Caribe de La Salle, otra institución más, que es la Universidad Experimental de Guayana fomenta la investigación antropológica. La antropología venezolana se fue apartando progresivamente de su inicial carácter comprometido con los temas sociales en la segunda mitad del siglo XX. Pero el ascenso de Hugo Chávez a la presidencia de la República marcará una fase nueva, caracterizada por la identificación de un importante grupo de antropólogos con los postulados políticos e ideológicos de la llamada revolución bolivariana, cuyo resultado más notable sería la nueva incorporación de la antropología a la participación en la transformación social de Venezuela.

En Ecuador y en Bolivia la institucionalización fue tardía, si se considera que no se visibiliza hasta los años setenta del siglo pasado. No obstante, muchas de las características que hallamos en las antropologías de Perú, Colombia y Venezuela, también las hallamos en la ecuatoriana y en la boliviana. En Ecuador la antropología nace en alguna medida bajo la influencia de los investigadores norteamericanos que trabajan en el país. Sin embargo, las primeras generaciones de antropólogos ecuatorianos, correspondientes a los años sesenta y setenta del siglo XX, se habían formado en el Centro de Investigaciones y Estudios Superiores en Antropología Social (CIESAS) de México, así como en la Universidad Iberoamericana, siendo Ángel Palerm la figura que lideraba ambas instituciones y la que adquirirá una singular influencia en la antropología ecuatoriana. Estos investigadores que habían adquirido sus conocimientos en México se unirían a los formados en los Estados Unidos, en Francia y en otros países, estimulando el progreso de la antropología en las últimas décadas del siglo XX. La Universidad Católica, desde 1970, y la Universidad Politécnica Salesiana se convirtieron muy pronto en los centros ecuatorianos por excelencia, desde los que se propagó la enseñanza de la antropología, así como la FLACSO desde 1991. Cristalizó de esta manera una antropología ecuatoriana, estrechamente unida a otras antropologías del Sur, como son la de Colombia, la de Ecuador y, quizá, antes que ninguna otra, a la de México, que ha convivido con la influencia muy notable de una antropología del Norte, que es la norteamericana, como explica Eleder Piñeiro. La antropología de Ecuador, emulando desde su nacimiento la antropología mexicana de la diversidad cultural, reforzará esta perspectiva con el fortalecimiento del punto de vista histórico, por influencia del propio Palerm, pero también de Wolf y de Mintz. Una característica más, propia no solo de la antropología ecuatoriana y de la boliviana, sino de otras antropologías latinoamericanas, vendrá dada por la importancia que adquiere la antropología aplicada, particularmente orientada al desarrollo, lo cual motivó la crítica de Worsley, Balandier y Meillassoux, al apoyar una antropología del desarrollo antes que una antropología para el desarrollo, como en su momento recordó A. Escobar (1999), y R. Pérez-Gañán analiza en su escrito sobre las antropologías de Ecuador y Bolivia. Por lo demás, la antropología ecuatoriana registraría muy pronto la influencia de Philippe Descola y de Anne C. Taylor, por razón de sus investigaciones ecuatorianas y de la significación que adquirieron ambos en la vida académica del país. La antropología ecuatoriana del presente se halla muy sensibilizada ante temas como el del género, la desigualdad, la educación, la sanidad, el extractivismo y otros, que no son ajenos al resto de las antropología de los países andinos, y, al igual que estas, se muestra especialmente apegada a la realidad social en la que se inserta, la cual nutre la sabia de su investigación.

Muchos de los rasgos definitorios de la antropología ecuatoriana los hallamos, asimismo, en la boliviana. Al igual que en el caso de aquélla, su institucionalización se produce en los años setenta del siglo XX, al socaire de los estudios de los antropólogos franceses, británicos, españoles, japoneses y norteamericanos que desarrollan sus investigaciones en el país. Numerosos antropólogos extranjeros, cuyas teorías han alimentado desde hace décadas el cuerpo científico de la antropología boliviana, destacando entre ellos J. Murra, W. Carter, T. Abercrombie, H. Buechler, J. Nash y otros, los cuales han realizado largos trabajos de campo en Bolivia, a la vez que han contribuido a alentar una producción realizada por los antropólogos bolivianos, la cual ha adquirido relevancia a partir de los años ochenta. La antropología boliviana ha privilegiado, sobre cualquier otro tema, el relativo a los estudios indigenistas, incluso en el siglo XXI, cuando en el resto de los países andinos habían dejado de ser una prioridad, en 
la senda de una tendencia que ha sido tradicional en Bolivia. Quizá este hecho explique la importancia que adquirido desde hace décadas una especie de confusión epistemológica que se produce entre la antropología y la historia, que lleva a los antropólogos a investigar el pasado prehispánico (como se evidencia desde los tiempos de Murra) y a los historiadores a examinar la etnogénesis (como sucede desde los primeros trabajos de Wachtel), tal como explica Anaïs Lallemant en su artículo. Es así que la antropología boliviana se ha ocupado, con distintos paradigmas y enfoques, de asuntos como el ayllu, la etnicidad, la reciprocidad, las mesas rituales, y, cada vez más, de otros, como la problemática rural/urbano, el género, la educación y el conflicto extractivista, que son comunes a las antropologías de los países andinos. Tampoco falta la perspectiva aplicada que se muestra en todos los países del área, e, incluso, el llamado giro ontológico que permite situar junto a los humanos a todo tipo de animales, plantas y cosas, en un contexto de descentración antropológica.

\section{Conclusión}

Mucho antes de que cristalizara la antropología académica en los países andinos, una ola de fervor indigenista recorría los encendidos textos de políticos e intelectuales. Al mismo tiempo que contribuían a la elaboración de los discursos sobre las identidades nacionales, estos escritores, de manera desigual según los países, iban abonando el fértil terreno en el que germinaría una antropología rica en matices. Nada más comenzar los años cuarenta del siglo XX se iniciaría el proceso de institucionalización en países como Perú y Colombia, y antes de que concluyera esa década también en Venezuela. Eran países que, ya por entonces, contaban con una sólida arquitectura universitaria que, complementariamente, estaba cada vez más abierta a las influencias extranjeras. La alta valoración que les merecen a los primeros antropólogos las culturas de sus países de nacimiento, en concurso con una débil infraestructura científica, carente generalmente de recursos suficientes para la investigación, Ileva a estos investigadores a realizar una meritoria antropología que, por regla general, se desarrolla dentro de las fronteras nacionales. Corriendo el tiempo, y, por razones análogas a las expuestas, en los años setenta del siglo pasado, se produciría la institucionalización de la antropología en Ecuador y en Bolivia.

Sin embargo, y a pesar de que la cordillera andina ha conferido una personalidad geográfica a estos países, y por más que todos poseen una historia compartida en muchos aspectos, y, a mayores, considerando que todos ellos están recorridos por el denominador común de la diversidad cultural, las diferencias entre sus antropologías son también muy notables. De igual manera que no existe una antropología latinoamericana, tampoco existe una andina. Cada una de estas antropologías andinas posee personalidad propia, pero no es menos cierto que también comparten rasgos; en primer lugar, porque están unidas por redes sociales e intelectuales internas, y, en segundo lugar, porque participan de intereses y preocupaciones comunes con el resto de las antropologías latinoamericanas. La alteridad no se construye a partir de un otro distante, sino que el otro que da pábulo a la epistemología antropológica es idéntico y cercano, tal y como predicaba la antropología crítica latinoamericana de los años setenta y ochenta del siglo XX. Ciertamente, la descolonización abocó al nacimiento de Estados nacionales caracterizados por una decidida exclusión de poblaciones que ni siquiera ha cesado en el presente por efecto de la permanente colonialidad que mostraba Quijano. Los antropólogos, de acuerdo con esta reflexión, no solo son miembros de esas sociedades, sino que su vivencia cotidiana no podría entenderse si no fuera como parte de una cotidianidad conflictiva, en la que los prejuicios, los estereotipos, la discriminación y la desigualdad son realidades indubitables. En estas condiciones, el otro que estudia el antropólogo no es uno diferente, sino que es uno mismo, encarnado en la vida de los estudiados. Por lo tanto, la teoría de la reflexión que se encuentra tras las antropologías del Sur, subalternas, nativas o segundas, comporta un cuestionamiento de esa alteridad que ha sido distintiva de la ciencia antropológica desde su mismo nacimiento.

Por su parte, los antropólogos más afectos al cultivo de una antropología que podemos llamar clásica, continuaron realizando sus investigaciones y estableciendo sinergias con la antropología crítica, de suerte que se produjo un enriquecimiento recíproco de los temas característicos de la antropología. La antropología es una reflexión sobre la otredad que progresa en riqueza conforme aumentan los puntos 
de vista de los observadores. Los paradigmas antropológicos son complementarios antes que excluyentes. A finales del siglo XX el panorama antropológico se hallaba muy renovado, de suerte que el tratamiento de la alteridad había adquirido una gran riqueza. Las antropologías de los países andinos de finales del siglo $X X$ y de los primeros lustros del siglo $X X I$, con una presencia creciente en la vida académica, revelan un gran interés por los colectivos étnicos, entre los que siguen estando presentes los indígenas, pero también por los afrodescendientes y otros grupos, por el cambio cultural, por las políticas de desarrollo, y por una diversidad de temas a los que se les había prestado escasa atención en el pasado, como las desigualdades de género, la educación, la salud, el medio ambiente (especialmente, los conflictos motivados por el aprovechamiento de los recursos extractivos), la interculturalidad, y tantos otros, a los que se han añadido los derivados del postconflicto y de la memoria histórica. Son antropologías con acento sujetas actualmente a una constante renovación teórica y metodológica, atentas al elemento humano con el que conviven, pero abiertas a las influencias exteriores, que son resultado de una poderosa interacción entre lo local y lo universal.

\section{Bibliografía}

Cardoso de Oliveira, Roberto

1988 Sobre o pensamento antropológico. Rio de Janeiro, Ediçoes Tempo Brasileiro.

1996 "La antropología latinoamericana y la "crisis" de los modelos explicativos: Paradigmas y teoría", Maguaré, no 11-12: 9-23.

1999 y 2000 "Peripheral anthropologies 'versus' central anthropologies", Journal of Latin American Anthropology, vol. 4 (2) y 5 (1): 10-30.

Cardoso de Oliveira, Roberto (y Guilhermo Rubén) (ed.)

1995 Estilos de antropología. Campinas, Editora Unicamp.

Escobar, Arturo

1999 El final del salvaje. Bogotá, Instituto Colombiano de Antropología e Historia y Centro de Estudios de la Realidad Colombiana.

Gadamer, George

1960 Verdad y método. Salamanca, Ediciones Sígueme, 1994.

Geertz, Clifford

1973 La interpretación de las culturas. Barcelona, Gedisa, 2005.

Gómez-Pellón, Eloy

2012 "Las premisas epistemológicas y la antropología social”, Gazeta de Antropología, $\mathrm{n}^{\circ} 28$ (1). https://dialnet.unirioja.es/servlet/articulo?codigo $=4282908$

Krotz, Esteban

1993 "La producción de la antropología en el Sur: características, perspectivas, interrogantes", Alteridades, vol. 3 (6): 5-11.

1997 "Anthropologies of the South: their rise, their silencing, their characteristics", Critique of Anthropology, vol. 17(3): 237-251.

2007 "Cuatro cuestiones cruciales para el desarrollo de nuestras antropologías", en A. Giglia, C. Garma y A. P. de Teresa (comp.), ¿Adónde va la antropología? México, Juan Pablos, Universidad Autónoma Metropolitana-Unidad Iztapalapa y Tecnigraf: 157-176.

2015 "Las antropologías segundas en América Latina: interpelaciones y recuperaciones", Cuadernos de Antropología Social, $\mathrm{n}^{\circ}$ 42: 5-17. 
Kuhn, Thomas

1962 La estructura de las revoluciones científicas. Bogotá, Fondo de Cultura Económica, 1992.

Lins Ribeiro, Gustavo (y Arturo Escobar) (ed.)

2008 Antropologías del mundo. Transformaciones disciplinarias dentro de sistemas de poder. Popayán, CIESAS, Envión, The Wenner-Gren Foundation.

Narotzki, Susana

2011 "Las antropologías hegemónicas y las antropologías del Sur: el caso de España", Revista Andaluza de Antropología, nº 1: 26-40.

Quijano, Aníbal

2000 "Colonialidad del poder, eurocentrismo y América Latina", en E. Lander (comp.), La colonialidad del saber: eurocentrismo y ciencias sociales. Perspectivas latinoamericanas. Buenos Aires, CLACSO: 777-832.

Restrepo, Eduardo

2014 "Antropología hecha en Colombia", Revista Antropologías del Sur, n 1: 83-104.

2016 Escuelas clásicas del pensamiento antropológico. Cuzco: Impresiones Gráficas.

Sousa Santos, Boaventura de (y Paula Meneses) (ed.)

2014 Epistemologías del Sur (perspectivas). Madrid, Akal.

Uribe, Carlos Alberto

1997 "A Certain Feeling of Homeless. Remarks on Estaban Krotz Anthropology of the South", Critique of Anthropology, vol. 17, $\mathrm{n}^{\circ}$ 3: 253-261. 